\title{
Ultrastructure of the placenta in gestational diabetes mellitus
}

\author{
Abdelghany Hassan, Tarek M. Essa \\ Department of Anatomy and Embryology, School of Medicine, Alexandria University, Alexandria, Egypt
}

\begin{abstract}
Objectives: The placenta plays critical roles during pregnancy and is essential for fetal growth and development. Its functions are determined by the ultrastructure of the placental barrier that is an important feature to maintain the exchange surface area between the fetus and the mother. Gestational diabetes mellitus (GDM) comprises unfit conditions for embryonic and feto-placental development, and may result in placental abnormalities. The aim of this study was to detect the ultrastructural changes of the placenta in women with GDM.

Methods: The placentas of 10 women with GDM without pregestational diabetics, hypertension and chronic diseases and 10 controls were studied. Six control women were delivered vaginally and the remaining cases by caesarian section at a gestational age of 36 to 39 weeks. Placental samples were measured for their thickness and prepared for light and transmission electron microscopy study.

Results: Light microscopic study of the control placentas showed numerous densely packed microvilli with syncytial knots and thin-walled blood vessels and wide intervillous spaces. The placentas of GDM cases showed reduced number of microvilli with syncytial knots, thick-walled vessels, edematous spaces, areas of fibrosis and perivillous fibrinoid degeneration. Electron microscopic study of the placentas of the control women showed terminal villi with a thick layer of syncytiotrophoblasts (Sy) with a lot of regular cylindrical microvilli and a thin layer of cytotrophoblasts (Cy). There were some endoplasmic reticulum cisternae besides few mitochondria. The underlying villus core was harboring fetal capillaries lined with flat endothelial cells and thin basement membrane. There was no fibrosis or edema. In the placenta of GDM women, there was hypertrophy of Cy with atrophy of Sy with multiple vacuoles and areas for glycogen storage. The subtrophoblastic membrane was thick and the microvilli were scarce. The villous core showed congested capillaries, stromal macrophages, edematous spaces, glycogen storage areas and fibrosis.

Conclusion: All the changes in placentas of gestational diabetes were attributed to associated hypoxia and oxidative stress due to decreased uteroplacental flow that was aggravated by the thick placental barrier and the presence of edema, fibrosis and glycogen storage areas that increased the distance of transfer between the fetus and mother.
\end{abstract}

Keywords: cytotrophoblast; fetal; gestational diabetes; microvilli; placenta; syncytiotrophoblast

Anatomy 2016;10(3):159-169 @2016 Turkish Society of Anatomy and Clinical Anatomy (TSACA)

\section{Introduction}

The placenta, situated between the mother and fetus, is essential for fetal growth and development. ${ }^{[1]}$ Placenta performs essential functions during pregnancy, including the exchange of nutrients, water, respiratory gases and waste products, and synthesis of various hormones which regulate the transport of maternal nutrients to the fetus and facilitate maternal metabolic adaptation to different pregnancy stages. These functions are determined by the ultrastructure of the placental exchange barrier. $^{[2]}$
The placental barrier that separates the fetal and maternal blood is composed of different structures including a continuous maternal-facing layer of syncytiotrophoblasts (Sy) with multiple apical microvilli, a thin layer of cytotrophoblasts $(\mathrm{Cy})$ which gets thinner as pregnancy proceeds but persists until term, the endothelial lining and underlying basal membrane (BM) of the fetal capillaries, and the villous connective tissue between them. This barrier is mostly important to maintain the exchange surface area between the fetus and the mother. ${ }^{[2,3]}$ A proper coordination of trophoblast proliferation, differentiation and invasion is required for placental development. ${ }^{[3,4]}$ 
The physiological changes that occur during pregnancy can only be sustained if there is an appropriate nutrient supply to ensure placental and fetal development. To make sure that the supply of nutrients is essential to fetal survival, the placenta continuously undergoes changes in weight, structure, shape and function during gestation. Some forms of intrauterine fetal growth abnormalities have been correlated with abnormalities of placental blood flow and transfer of nutrients from the mother to the fetus. ${ }^{[1]}$

Gestational diabetes mellitus (GDM) is defined as the glucose intolerance with onset or first recognition during pregnancy. GDM is associated with short- and long-term morbidity in both offspring and mother. The short-term adverse outcomes include macrosomia, neonatal hypoglycemia, neonatal jaundice, preeclampsia, preterm delivery and cesarean delivery, while the long-term complications include obesity, abnormal glucose tolerance, and diabetes in adolescence or early adulthood. ${ }^{[5,6]}$

The diabetic environment can be regarded as a network of substances (hormones, nutrients, cytokines) with altered concentrations. ${ }^{[7]}$ In diabetes, the placenta undergoes a variety of structural and functional changes. The nature and extent of these changes depend on a range of variables including the quality of glycemic control achieved during the critical periods in placental development and the modality of treatment. ${ }^{[7,8]}$

Despite currently available treatment, maternal diabetes comprises unfit conditions for embryonic and fetoplacental development and placental structure. However, reports on the pathology of placenta are usually incompatible with diabetes mellitus. The inconsistency may be explained partially by the fact that the category of diabetic pregnant women is not homogeneous. Moreover, the analysis of placental lesions in maternal diabetes has been complicated by superimposed hypertensive and other associated complications. ${ }^{[1]}$

Few studies have investigated the ultrastructural changes in human term placentas in GDM. ${ }^{[2]}$ Therefore, this study was undertaken to detect the ultrastructural changes of the placenta in women with GDM.

\section{Materials and Methods}

The placentas of 20 women, 10 control and 10 with GDM, were studied with free consents approved by the Committee of Ethics in Alexandria University, School of Medicine. Women with a history of pregestational diabetes, hypertension or other chronic diseases were excluded. The age of the women ranged from 28 to 30 years for the controls and 27 to 31 years for the GDM group. Fasting blood glucose level was measured after an overnight fast of at least $10 \mathrm{~h}$ at 24-28 weeks of gestation. The diagnosis of GDM was made based on the criteria of the American Diabetes Association (2011) ${ }^{[5]}$ Control women were within the accepted normal range of blood glucose levels from 90 to $115 \mathrm{mg} / \mathrm{dL}$ throughout gestation, while the GDM women showed levels ranging from 120 to $180 \mathrm{mg} / \mathrm{dL}$. GDM women were asked to control their diet besides insulin therapy, so that their fasting blood glucose levels were kept in the 100 to $135 \mathrm{mg} / \mathrm{dL}$ range until delivery. Two GDM women had higher fasting blood glucose ranging from 140 to $165 \mathrm{mg} / \mathrm{dL}$ due to poor diet control and interrupted therapy. The maternal weight at gestation ranged form 62 to $65.5 \mathrm{~kg}$ for the control, and from 65 to $68.5 \mathrm{~kg}$ for GDM group. All GDM women were delivered by caesarian section and the gestational weeks at delivery ranged from 36 to 38 weeks for the GDM. Six control women were delivered vaginally, while four were delivered by caesarian section at a gestational age ranging from 36 to 39 weeks. All deliveries were done at the Department of Gynecology and Obstetrics, Alexandria University, School of Medicine. The fetal birth weight ranged from 2.8 to 3.15 $\mathrm{kg}$ in the control group, while it ranged from 3.21 to $3.4 \mathrm{~kg}$ in the GDM group. The embryonic membranes and the umbilical cords were trimmed from the placentas. The placenta weight ranged from 450 to $480 \mathrm{~g}$ in the control group, and 470 to $500 \mathrm{~g}$ in the GDM group. The whole thickness of the placenta was measured at the center of the placenta, beside the site of attachment of the umbilical cord. Paraffin-embedded blocks from formaldehyde-fixed tissues were prepared and cut on a microtome at a thickness of $0.5 \mu \mathrm{m}$ from each block and stained with hematoxyline and eosin (HE) to be examined under Optika B-150 (Optika SRL, Ponteranica, Italy) light microscope. Blocks for electron microscopy were fixed in the fixative $3 \%$ glutaraldehyde and $2 \%$ paraformaldehyde in $0.1 \mathrm{~mol} / \mathrm{l}$ cacodylate buffer $(\mathrm{pH} 7.3)$ for $24 \mathrm{~h}$ at $4^{\circ} \mathrm{C}$. After fixation in $1.0 \%$ $\mathrm{OsO}_{4}$ in $0.1 \mathrm{~mol} / \mathrm{l}$ cacodylate buffer $(\mathrm{pH} 7.3)$ for $2 \mathrm{~h}$ at room temperature, the tissue specimens were subjected to dehydration in graded ethanol series. After immersion in propylene oxide (three times for $10 \mathrm{~min}$ each), the samples were immersed overnight in a mixture (1:1) of propylene oxide and Epon 812 resin (Sigma Aldrich, St. Louis, MO, USA) to be finally embedded in Epon-812 resin Semithin sections $(0.5 \mu \mathrm{m}$ thick) were cut using an ultramicrotome (Leica Ultracut; Leica, Berlin, Germany) that were picked on copper grids and counterstained with $2 \%$ uranyl acetate and lead citrate..$^{[9]}$ The specimens were then examined by transmission electron microscopy JEM-100CXi, (Jeol, Tokyo, Japan) at the electron microscope unit of Alexandria University School of Science.

The results were analyzed statistically using SPSS 17 (SPSS Inc, Chicago, IL, USA). Mean \pm standard deviation (SD) of the two groups were compared using paired-samples t-test and the level of significance was accepted as $\mathrm{p}<0.05$. 


\section{Results}

There was no statistically significant difference for the increase in gestational age in the control women compared to the GDM. Also, the higher maternal age in GDM women compared to the control was not statistically significant ( $p>0.5$ ). On the other hand, the maternal weight, neonatal weight and plasental weight were significantly increased in the GDM compared to controls (Table 1).

Light microscopy of the control placenta showed a large number of densely packed microvilli. The intervillous spaces were narrow and explicit and filled with blood cells. The microvilli showed syncytial knots which are aggregations of the nuclei of the Sy occupying vari-
Table 1

Parameters of the studies control and GDM cases.

\begin{tabular}{lcccc}
\hline Variables & Control & GDM & t-test & p value \\
\hline Gestational age & $36.9 \pm 0.91$ & $36.4 \pm 0.6$ & 1.88 & 0.076 \\
Neonatal weight & $2.93 \pm 0.127$ & $3.15 \pm 1.91$ & 5.955 & $0.001^{*}$ \\
Maternal age & $28.85 \pm 0.88$ & $29.20 \pm 1.36$ & 0.941 & 0.358 \\
Maternal weight & $63.97 \pm 1.14$ & $65.64 \pm 0.53$ & 5.807 & $0.001^{*}$ \\
Placental weight & $460.25 \pm 8.96$ & $485.25 \pm 11.47$ & 6.216 & $0.001^{*}$ \\
\hline
\end{tabular}

*Significant at $p<0.05$

able areas at the poles of the microvilli. The villous core showed small and thin-walled blood vessels showing a number of blood cells (Figure 1). In placenta of GDM,
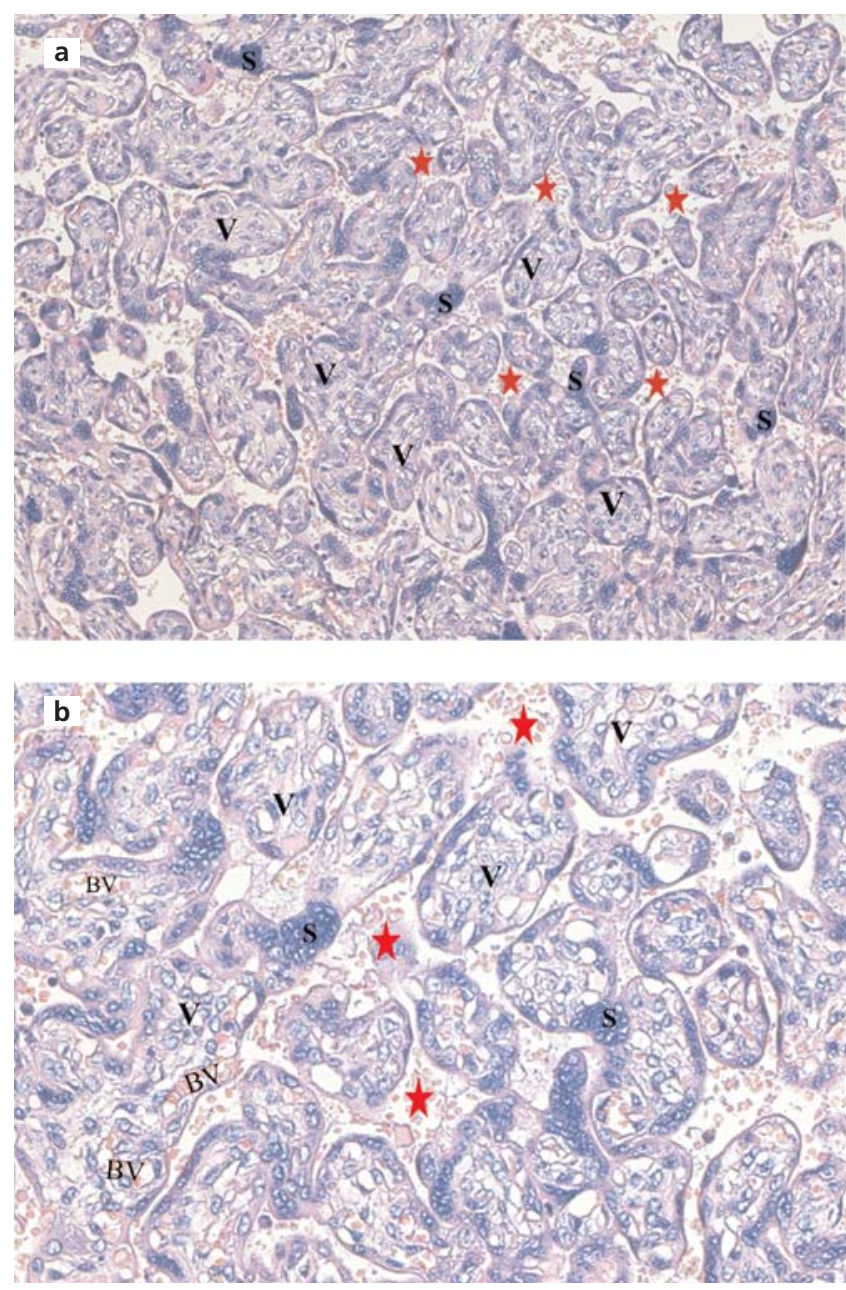

Figure 1. (a, b) Photomicrographs of the control placenta showing numerous microvilli $(\mathrm{V})$ with narrow intervillous spaces (red star) filled with blood cells. The microvilli show syncytial knots (S) at their poles. The villous core shows thin-walled blood vessels (BV) containing blood cells. Haematoxylene and eosin stain, $\times 200$ (a); $\times 400$ (b). [Color figure can be viewed in the online issue, which is available at www.anatomy. org.tr]
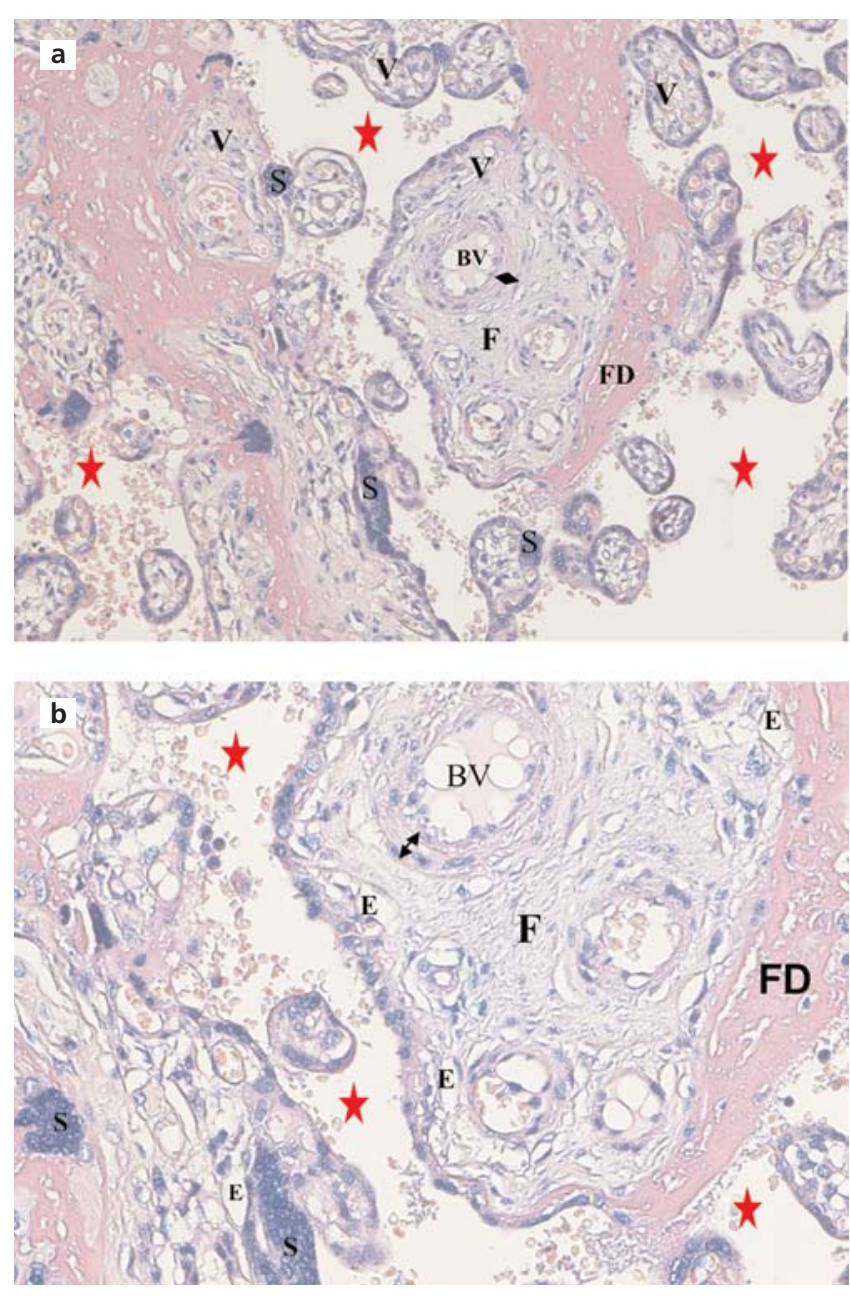

Figure 2. (a, b) A photomicrograph of GDM placenta showing reduced number of microvilli $(\mathrm{V})$ showing syncytial knots (S) with wide intervillous spaces (red star) filled with blood cells. The villous core shows areas of fibrosis (F), spaces of edema (E) and blood vessels (BV) containing blood cells and their walls (black arrow) were thick. There were areas of perivillous fibrinoid degeneration (FD). Haematoxylene and eosin stain, $\times 200$ (a); ×400 (b). [Color figure can be viewed in the online issue, which is available at muw.anatomy.org.tr] 


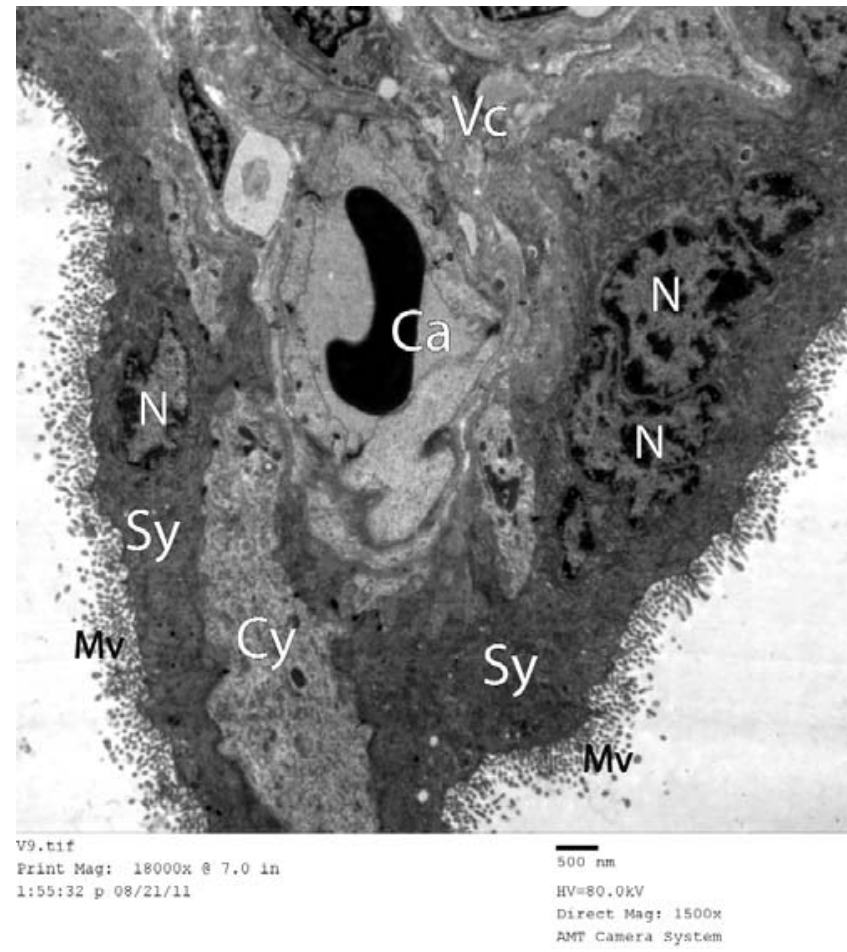

Figure 3. An electron micrograph of a terminal chorionic villus of a placenta from a control woman showing that the covering trophoblastic layer is formed basically of Sy that exhibits a relatively dense cytoplasm surrounding multiple nuclei $(\mathrm{N})$, with a lot of long cylindrical microvilli (Mv) on the surface. The underlying villus core (Vc) is harboring a capillary (Ca). Cytotrophoblast (Cy). Magnification $\times 1500$, Scale bar $=500 \mathrm{~nm}$.

the number of microvilli was less than the controls with syncytial knots. The intervillous spaces were wide. A number of the microvilli showed areas of fibrosis and scattered spaces of edema in the villous core. The blood vessels in some of the micovilli were dilated and showed thickening of their walls. There were areas of perivillous fibrinoid degeneration shown in bright pink appearance (Figure 2).

Electron microscopic study of the full-term placenta of control women showed that the terminal chorionic villi had a covering of a thick layer of Sy which had a lot of cylindrical and regular microvilli (Figures 3 and 4). The cytoplasm of Sy was relatively dense surrounding multiple nuclei with rough endoplasmic reticulum (rER) and mitochondria (Figures 3-6). Deeper to SY there was a thin layer of $\mathrm{Cy}$ where the cytoplasm of its cells was paler and harbored some rER cisternae beside few mitochondria (Figures 4 and 6). In some areas of the villi, there was only Sy, but no Cy (Figures 3 and 5). The underling villous core was harboring fetal capillaries that were lined with flat endothelial cells held together by dense junctions (Figures $\mathbf{5}$ and $\mathbf{7}$ ). The BM was too thin

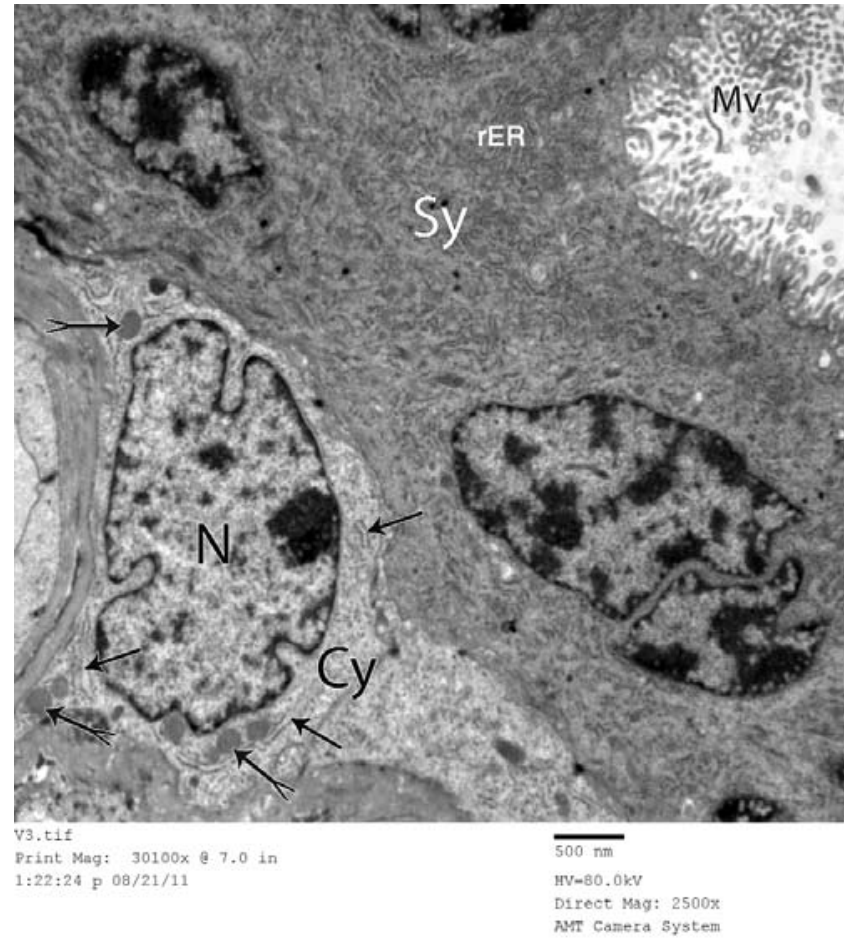

Figure 4. A higher Electron micrograph of a terminal chorionic villous of a control placenta. The Sy has its cytoplasm rich in rER profiles and bears slender and regular microvilli (MV). The Cy forms a thinner deep layer; the cytoplasm of this cell seemed paler and harbored some rER cisternae (arrows) beside few mitochondria (forked arrows). Magnification $\times 2500$, Scale bar $=500 \mathrm{~nm}$.

to be resolved. The capillaries were externally limited by fenestrated concentric arrays of reticular fibers (Figures 5 and 7 ).

In the placenta of GDM women, there was hypertrophy of Cy with atrophy of the Sy (Figure 8). The cytoplasm of both types of trophoblasts was occupied by a lot of membrane-bound irregular vesicular structures that contained a low electron-dense material (Figures 8-10). Areas rich in glycogen granules and few mitochondria appeared in the Cy cells (Figure 9). In the Sy, there were inclusion bodies inside the nucleus that assumed a concentric lamellar appearance with a halo around (Figure 10). The microvilli on the surface were scarce and some had terminal club ends (Figure 10). The subtrophoblastic BM was thick and separated from the fetal capillary by a subtrophoblastic space (Figure 9). The fetal capillaries underneath the trophoblast were congested with much dark and few pale red blood cells (RBCs) (Figure 8). The endothelial cells lining the capillaries showed aggregates of glycogen granules near the nucleus together with the presence of dilated cisternae of endoplasmic reticulum (Figure 11). There were edematous clear 


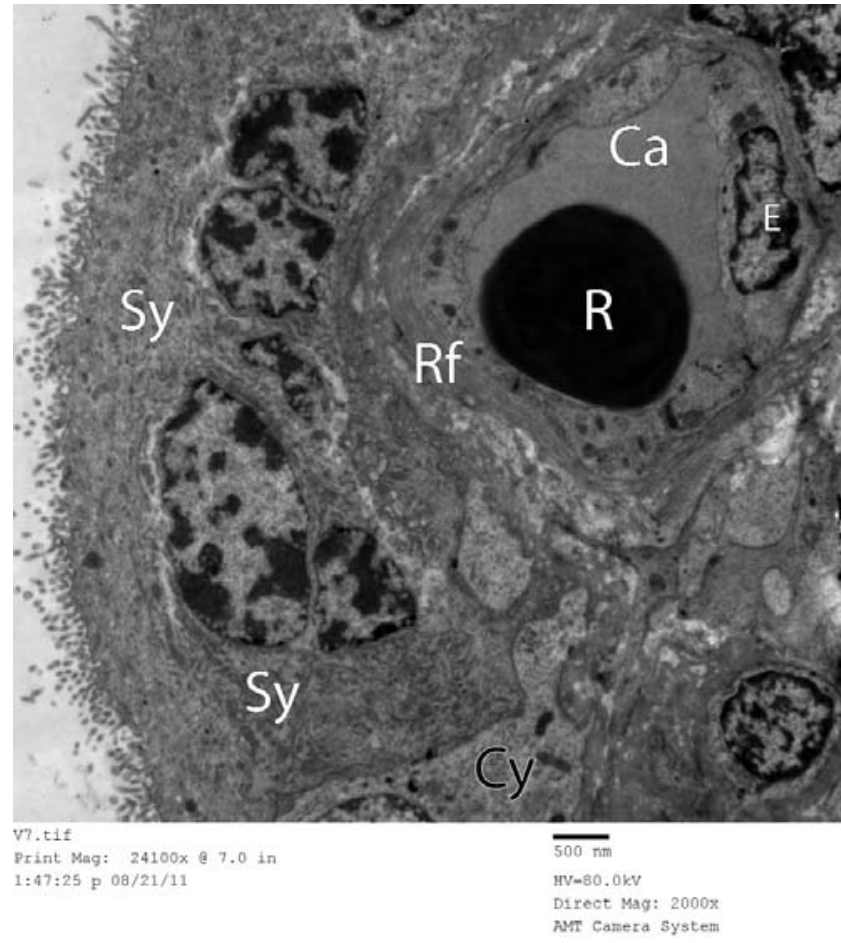

Figure 5. Electron micrograph of a terminal chorionic villous of a control placenta showing predominance of Sy and almost complete exclusion of the $\mathrm{Cy}$ in the placental barrier. The fetal capillary $(\mathrm{Ca})$ is lined by flat endothelium $(\mathrm{E})$ that is surrounded by multiple layers of reticular fibers (Rf). Fetal RBC (R). Magnification $\times 2000$, Scale bar $=500 \mathrm{~nm}$.

spaces and well-defined storage areas in the matrix of the villous core (Figures 12 and 13). These storage areas were well-circumscribed by microcapsules of coarse matrix fibers and enclosed scattered dense short fibrillar and granular content on a homogenous background of moderate electron density (Figure 13). There was fibrosis inside a chorionic villous core where fibroblasts and collagenous bundles were exceptionally prevalent (Figure 14). Stromal macrophages were observed with lysosomes containing a material with a density comparable to that of hemoglobin of the extra-vasated fetal RBCs (Figure 12).

\section{Discussion}

The placenta is a complex organ that fulfills vital roles during fetal growth. Because of its unique position, the placenta is exposed to changes in both maternal and fetal environments. The diabetic environment can be regarded as a network of nutrients and hormones. Diabetes affects both maternal and fetal environments with multiple effects on different body organs including the placenta that shows alterations from non-diabetic placenta. ${ }^{[7]}$

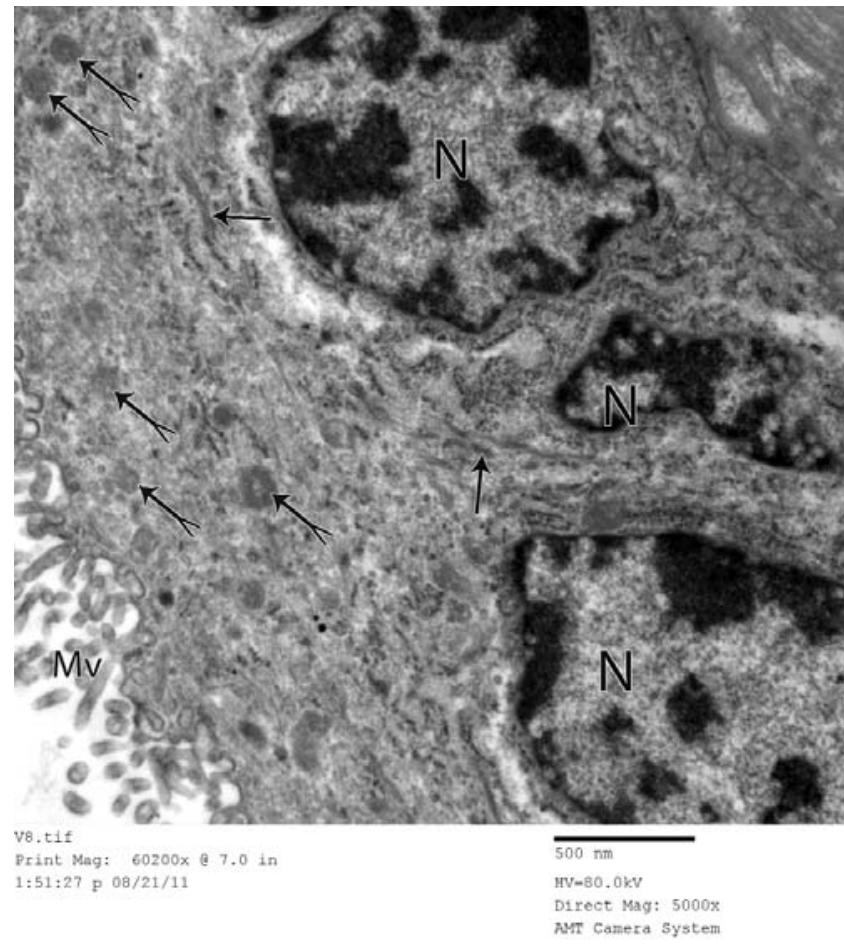

Figure 6. A magnified part from Figure 3 to reveal the ultra-structure of the Sy; rER (arrows), mitochondria (forked arrows), microvilli (Mv) and multiple nuclei $(\mathrm{N})$. Magnification $\times 5000$, Scale bar $=500 \mathrm{~nm}$.

Study of the placenta of the control group showed that the placental terminal villi were formed of a thick layer of Sy with multiple nuclei and a thin layer of $\mathrm{Cy}$ with a thin BM. The trophoblast showed some rER and mitochondria. Terminal villi contained regular and cylindrical microvilli. The villous core contained capillaries lined with flat endothelial cells with a thin BM with no evidence of fibrosis or edematous spaces. Meng et al. ${ }^{[2]}$ observed the same findings for the trophoblastic covering and the core of the placental villi.

Placental growth is essentially a result of the coordination of trophoblast proliferation and differentiation. The microvilli projecting from the Sy and their terminal branches play an important role in the fetal-maternal exchange. The thickness of the trophoblastic layers determine the microvillous density and the degree of trophoblastic maturation. ${ }^{[2]}$

In the control group, a thin layer of $\mathrm{Cy}$ is shown due to proper syncytial fusion. Sy cannot grow by itself, but syncytial growth and surface expansion throughout pregnancy together with endometrial invasion depend on continuous incorporation of $\mathrm{Cy}$ by syncytial fusion. This 


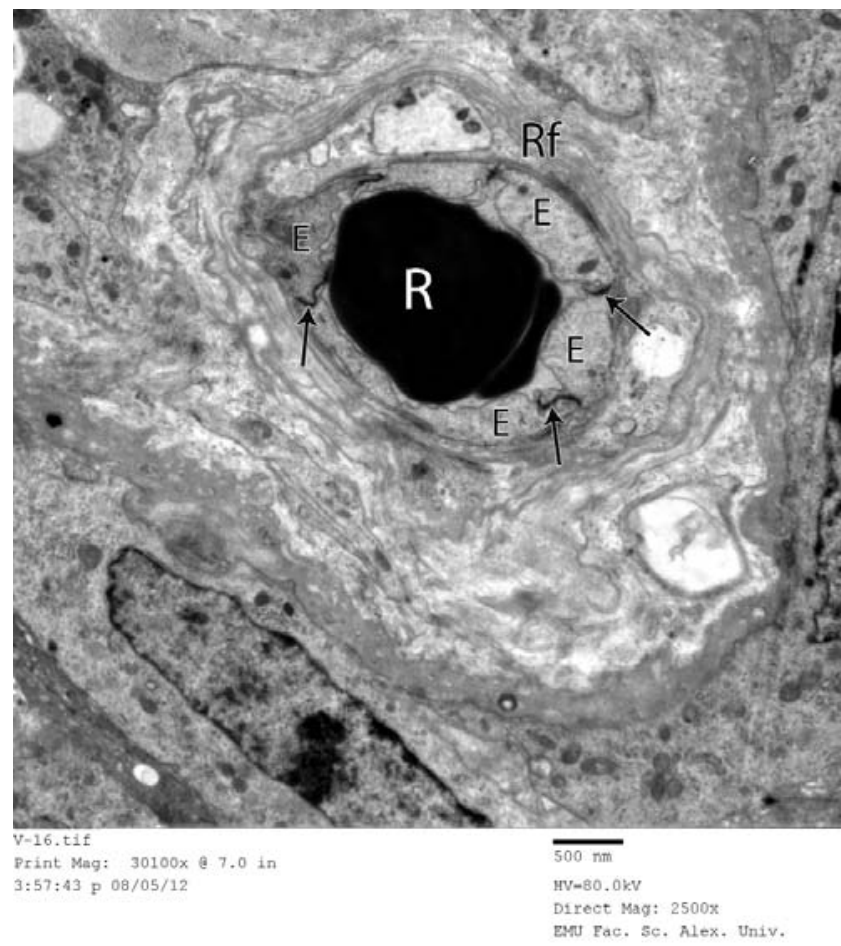

Figure 7. Detailed ultrastructure of a fetal capillary from a control placenta. This micro-vessel is lined by flat endothelial cells (E) that are held together by dense junctions (arrows). The BM is too thin to be resolved; however, the capillary is externally limited by fenestrated concentric arrays of reticular fibers (Rf). Fetal RBCs (R). Magnification $\times 2500$, Scale bar $=$ $500 \mathrm{~nm}$.

fusion is also important for the syncytial hormonal function. ${ }^{[4]}$ Thin $\mathrm{Cy}$ and hence the placental barrier increase the utero-placental blood flow into the intervillous spaces, thus ensuring adequate maternal nutrient supply to the fetus. ${ }^{[2,4]}$ Moreover, thin Cy cells ensure proper invasion to the endometrium and its spiral arteries as the $\mathrm{Cy}$ at the tips of the villi grows out to penetrate into the decidualised uterus. ${ }^{[4]}$ Placental invasion and establishment of enough maternal blood supply are key processes in placental development, their dysregulation is associated with pregnancy diseases. ${ }^{[2,3]}$

The placental barrier represents the site of interface between the maternal and fetal circulations. In the control group, it was formed of Sy, a thin layer of $\mathrm{Cy}$ and a thin layer of vascular endothelial cells with a thin BM together with a villous core made up of stroma without edema or fibrosis. Gude et al..$^{[10]}$ and Meng et al. ${ }^{[2]}$ stated that this structure is suitable to ensure proper oxygenation and nutrition of the embryo as proper oxygenation of the placenta and the embryo is essential for a successful pregnancy. ${ }^{[2,10]}$

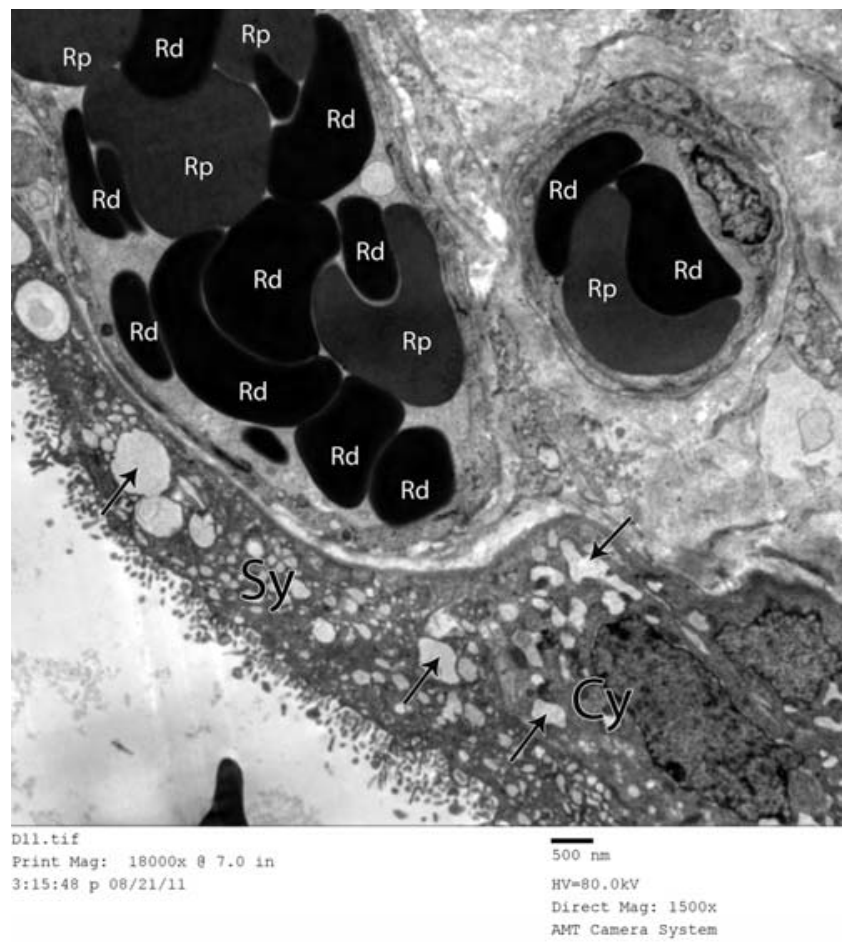

Figure 8. Electron micrograph of a terminal chorionic villous of a placenta from a woman with GDM. Notice hypertrophy of $\mathrm{Cy}$ on the expense of the atrophic Sy; the cytoplasm of both is occupied by dilated membrane-bound vesicular structures (arrows). The capillaries underneath the trophoblast are congested with much dark (Rd) and few pale $(\mathrm{Rp})$ fetal RBCs inside. Magnification $\times 1500$, Scale bar $=500 \mathrm{~nm}$.

The rER is the intracellular organelle where synthesis of proteins occurs prior to its eventual extrusion into the extracellular matrix to share in the production of placental hormones. Mitochondria are double-membrane organelles with multiple essential functions, such as cellular survival, energy metabolism, and intracellular adenosine triphosphate (ATP) production by oxidative phosphorylation necessary for the active organ. Oxidative phosphorylation is a highly efficient process in which cells use enzymes to oxidize nutrients, thereby releasing energy which is used to form ATP. Under normal conditions, increased activity and oxidative phosphorylation of placental mitochondria throughout gestation result in overproduction of reactive oxygen species (ROS) such as superoxide and hydrogen peroxide as natural by-products of the normal metabolism of oxygen. Physiological ROS levels play a crucial role in placental development through cellular signaling, but increased ROS can inactivate certain enzymes thus expose the placenta to oxidative stress even in normal pregnancies. This is dealt with by the placental antioxidant defense capacities which induce conversion of ROS to water and molecular oxygen..$^{[1,12]}$ 


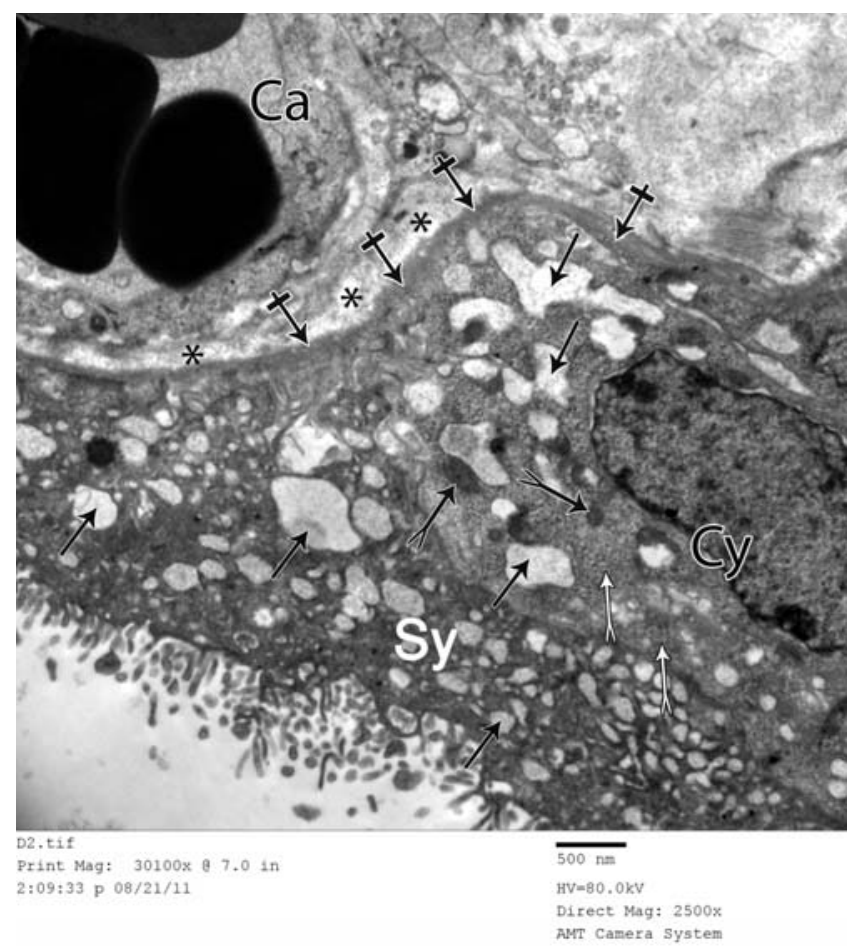

Figure 9. Higher magnification of Figure 6; the cytoplasm of both types of trophoblast is occupied by a lot of membrane-bound irregular vesicular structures (black arrows) that contain a low electron-dense material. Areas rich in glycogen granules (white forked arrows) and few mitochondria (black forked arrows) appear in the Cy cell (Cy). The sub-trophoblastic BM (crossed arrows) is obviously thick and is separated from the fetal capillary $(\mathrm{Ca})$ by a sub-trophoblastic space $\left(^{*}\right)$. Magnification $\times 2500$, Scale bar $=500 \mathrm{~nm}$.

The present study on placentas from women with GDM showed that the Sy was thin with thick Cy and scarce microvilli. Similar observations were found by Meng et al., ${ }^{[2]}$ Gheorman et al. ${ }^{[8]}$ Slukvin et al.$^{[13]}$ and Gul et al. ${ }^{[1]}$ who found reduced number of or even absent placental microvilli with immature villous Sy and persistent Cy with GDM.

It has been suggested that diabetes induces abnormal uncontrolled cell proliferation in the placenta similar to that seen in other organs, with increased proliferative activity in villous $\mathrm{Cy} .^{[, 1,1]}$ This adds to the uteroplacental ischemia and hypoxia as the cells of the thick Cy layer at the tips of the villi do not invade properly to the endometrial vessels. This strongly suggests an influence of the maternal diabetic environment on trophoblastic invasion. Invasion is a complex process involving a range of invasion inhibiting and invasion promoting factors. The diabetic environment appears to shift the balance towards invasion inhibition as a response to accompanying diabetic oxidative stress. ${ }^{[1,15]}$ This shallow invasion

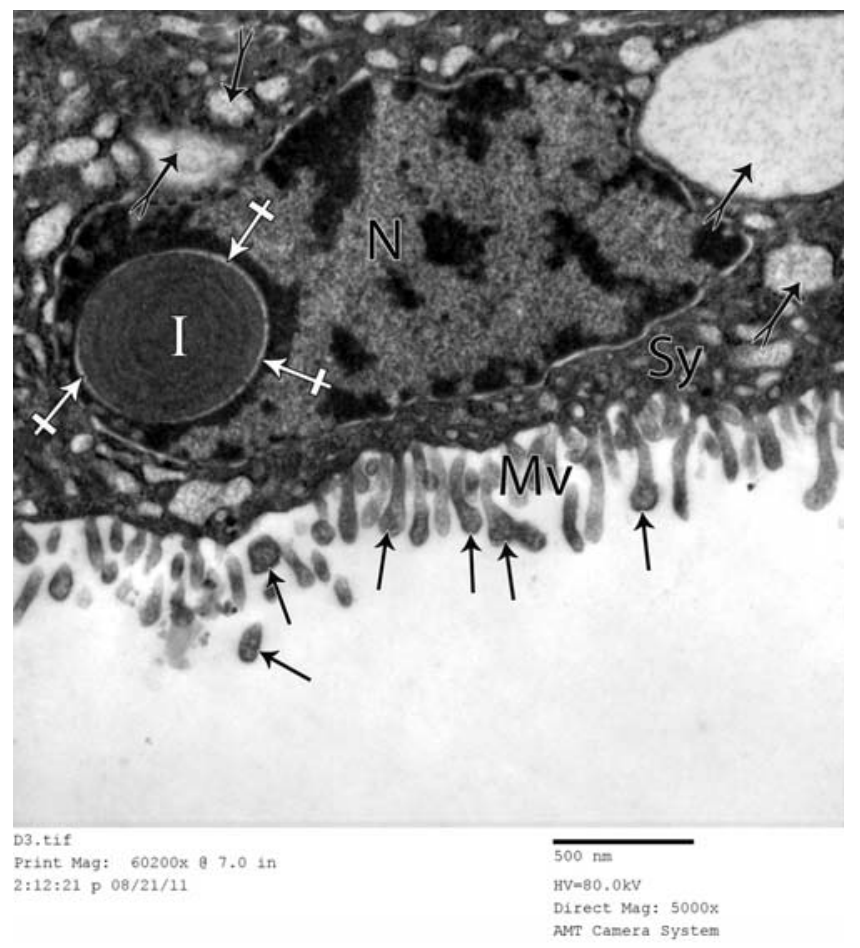

Figure 10. Electron micrograph of Sy in the placenta in a woman with GDM. It demonstrates the appearance of an inclusion body (I) inside the nucleus (N); it assumes a concentric lamellar appearance with a halo around (crossed arrows). Microvilli (Mv) on the surface are scarce and some have terminal club ends (black arrows). There are cytoplasmic vesicular structures (forked arrows). Magnification $\times 5000$, Scale bar $=$ $500 \mathrm{~nm}$.

reduces the uteroplacental blood flow resulting in hypoxia, increased placental infarction areas and Sy cell degeneration with shedding and reduction of the number of the villi. ${ }^{[2,13]}$ It was suggested that villous Cy proliferation without syncytial fusion, as observed in severe hypoxia, might be accompanied by syncytial degeneration. $^{[3]}$

Oxidative stress and mitochondrial dysfunction are now emerging as a front- runner in the mechanism of diabetic pathogenesis. Recently, a two fold increase in trophoblastic mitochondrial ROS production through incomplete reduction of oxygen under hyperglycemic conditions when compared to normoglycemic levels with the reduction of antioxidant capacity was reported. ROS causes a biological molecular damage leading to inactivation of specific enzymatic reactions together with loss of function and cell death with the resulting oxidative stress. Thus, the trophoblasts are affected by the hyperglycemic environment with reduction of its proliferation. ${ }^{[16-18]}$ 


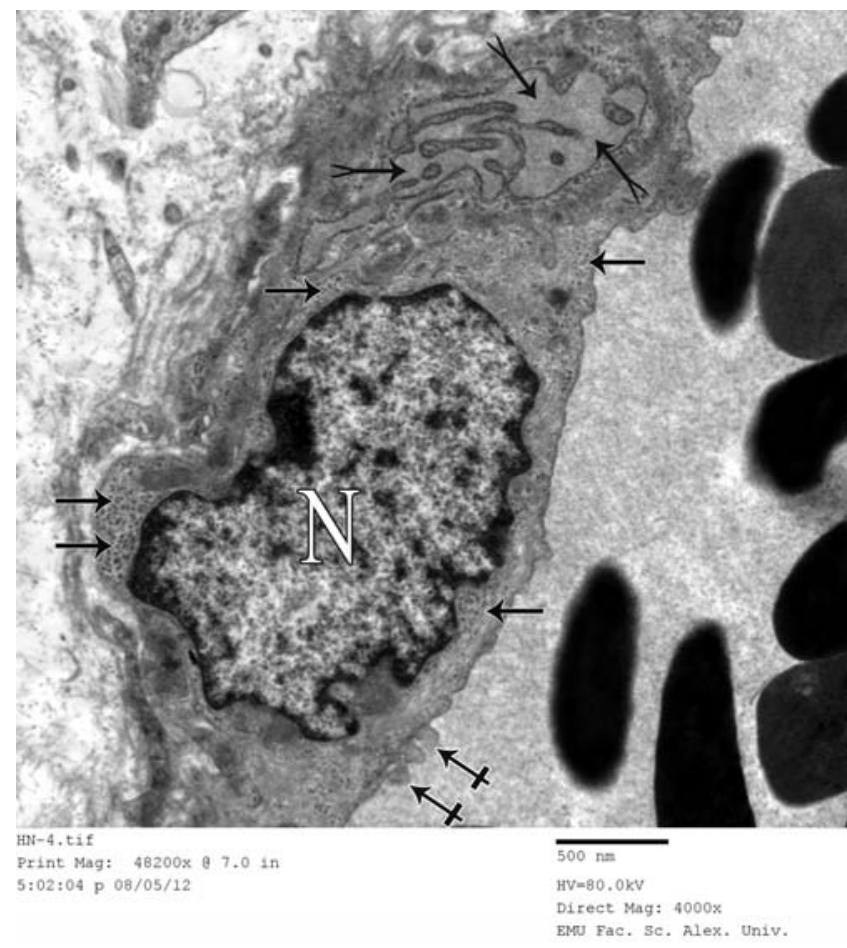

Figure 11. Electron micrograph with very high power magnification of a fetal capillary in a woman with GDM. Aggregates of glycogen granules (arrows) are stored particularly near the nucleus (N) of an endothelial cell. Also, dilated cisternae of endoplasmic reticulum (forked arrows) are shown where a homogeneous material of moderate electron density is stored. Notice blebbings of capillary endothelial cytoplasm into the capillary lumen (crossed arrows). Magnification $\times 4000$, Scale bar $=500 \mathrm{~nm}$.

The present study showed thick trophoblastic BM in diabetic placentas. Similar results were obtained by Gheorman et al., ${ }^{[8]}$ Magee et al. ${ }^{[19]}$ and Meng et al. ${ }^{[2]}$ who found thick BM of the villous trophoblast in women with GDM. The same was found by Gul et al. ${ }^{[1]}$ in the placenta of diabetic rats. BM thickening might be attributed to mucopolysaccharide storage and impaired villous trophoblastic activity such as increased production or decreased turnover of $\mathrm{BM}$ molecules, as it is known that the constituents of the $\mathrm{BM}$ components are produced by the secretion of trophoblasts. ${ }^{[8]} \mathrm{BM}$ thickening is also attributed to fat droplet accumulation due to an effect of diabetes on fatty acid oxidation. ${ }^{[1,13]}$ These changes may result in uteroplacental vascular insufficiency further aggravating the hypoxic state. ${ }^{[1]}$

Also, the current study showed the presence of vacuoles in both layers of the trophoblasts. It was hypothesized that a slight vacuolation may be present in the normal human placenta as a natural and physiological form of cell degradation which promotes survival. ${ }^{[2,21]}$ Meng et al. ${ }^{[2]}$ found much more trophoblastic vacuoles in placen-

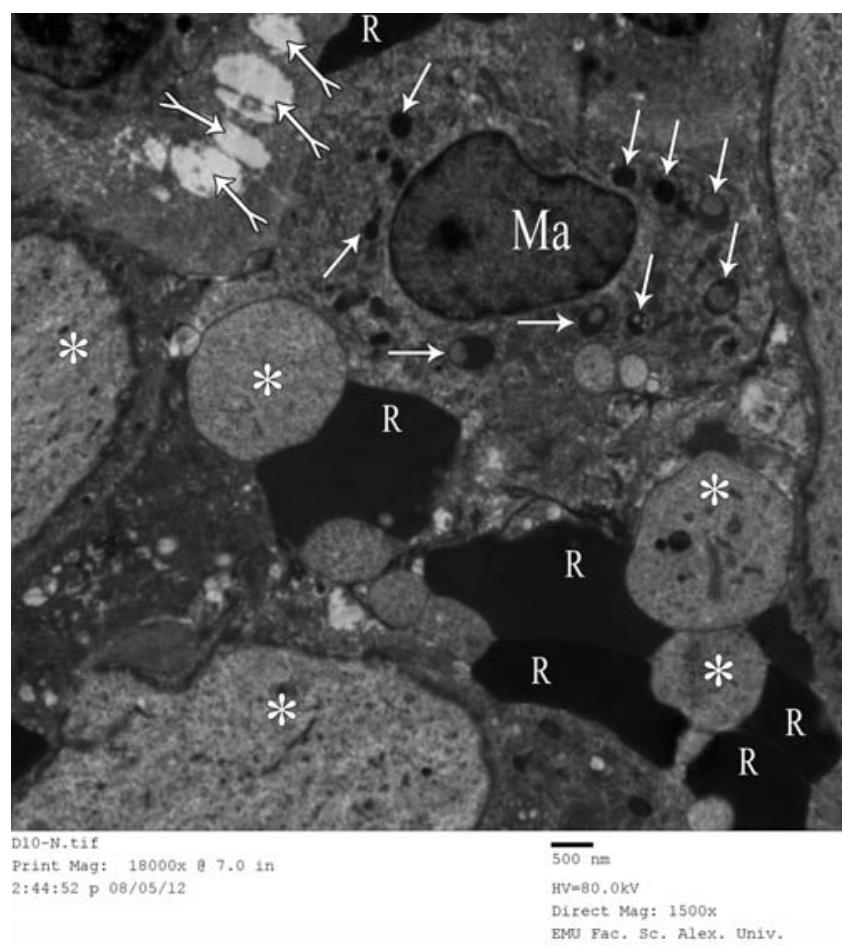

Figure 12. Electron micrograph of the core of a terminal chorionic villus of a woman with GDM. Notice, edematous clear spaces (forked arrows) and well-defined storage areas $\left(^{*}\right)$ inside the villous core matrix. A stromal macrophage $(\mathrm{Ma})$ is seen that contains lysosomes (arrows) with a dense material comparable to that of hemoglobin of the extra-vasated fetal RBCs (R). Magnification $\times 1500$, Scale bar $=500 \mathrm{~nm}$.

tas of GDM compared to controls. ${ }^{[2]}$ Hyperglycemia and hypoxia which have a role in the etiology of GDM may enhance the lysosome/vacuole functions of the trophoblasts, which result in widespread cytoplasmic vacuolation and altered transplacental metabolic exchange that may lead to cell death. So, in the context of disease, vacuolation is observed as a response to stress and oxygen restriction. ${ }^{[2,21,22]}$

Regarding the villous core, the present study showed that edematous spaces were obviously seen in women with GDM. Gauster et al. ${ }^{[23]}$ and Slukvin et al. ${ }^{[13]}$ mentioned that the placental villi showed spaces consistent with villous edema in GDM women. The same was found by Gul et al. ${ }^{[1]}$ on diabetic rat placentas. Gheorman et al. ${ }^{[8]}$ attributed the villous edema to the mucopolysaccharide deposits that consisted mainly of hyaluronic acid molecules that can retain water. This edema fluid is interposed as a barrier to the exchange between the mother and fetus. ${ }^{[1]}$

Also, the present study showed dilatation of the endoplasmic reticulum in the cytoplasm of the vascular 


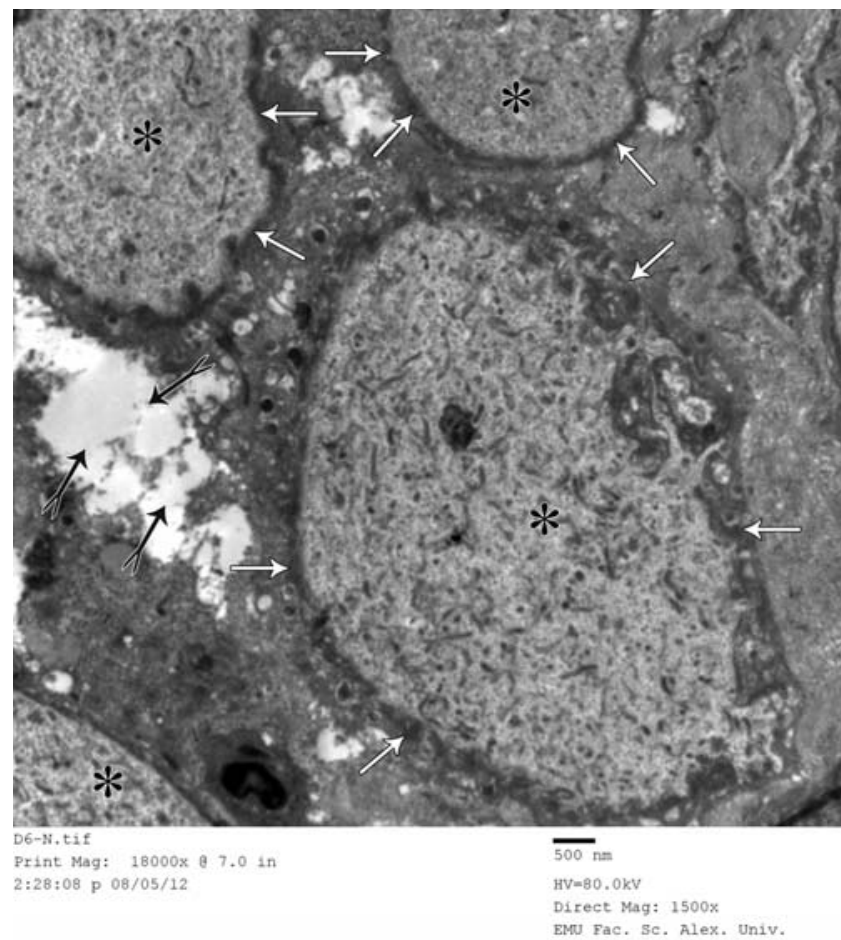

Figure 13. Electron micrograph of the core of terminal chorionic villous of a woman with GDM, showing the detailed structure of the storage areas $\left(^{*}\right)$. These areas are well-circumscribed by micro-capsules (arrows) of coarse matrix fibers and enclose scattered dense short fibrillar and granular content on a homogenous background of moderate electron density. Notice, edematous clear spaces (forked arrows). Magnification $\times 1500$, Scale bar $=500 \mathrm{~nm}$

endothelial cells. Same findings were observed by Meng et al. ${ }^{[2]}$ and Gul et al. ${ }^{[1]}$ on diabetic rats. rER is one of the most vulnerable organelles susceptible to hyperglycemia and hypoxia. ${ }^{[12]}$ Massively dilated rER, however, is an ultrastructural indication of improper processing associated with a disorder that prevents proper folding and extrusion of protein products, and this was attributed to oxidative stress induced cell injury in diabetes. ${ }^{[1,24]}$ The abnormal ultrastructure of rER could have impacts on metabolic functions and synthesis in the placenta. ${ }^{[2]}$

Also, the present study showed areas of glycogen storage inside the trophoblast and villous core. Gheorman et al. ${ }^{[8]}$ showed the presence of glycogen storage areas in placentas of diabetic mothers. Gul et al., ${ }^{[1]}$ Yoruk et al..$^{[25]}$ and Padmanabhan and Shafiullah ${ }^{[26]}$ found increased glycogen content in the placental villi in diabetic rats. This glycogen deposition around the villous capillaries was related to the extent of maternal hyperglycemia that caused increased insulin levels in the fetal circulation. Fetal hyperinsulinemia stimulates what is called the buffer action of the placenta by stimulating

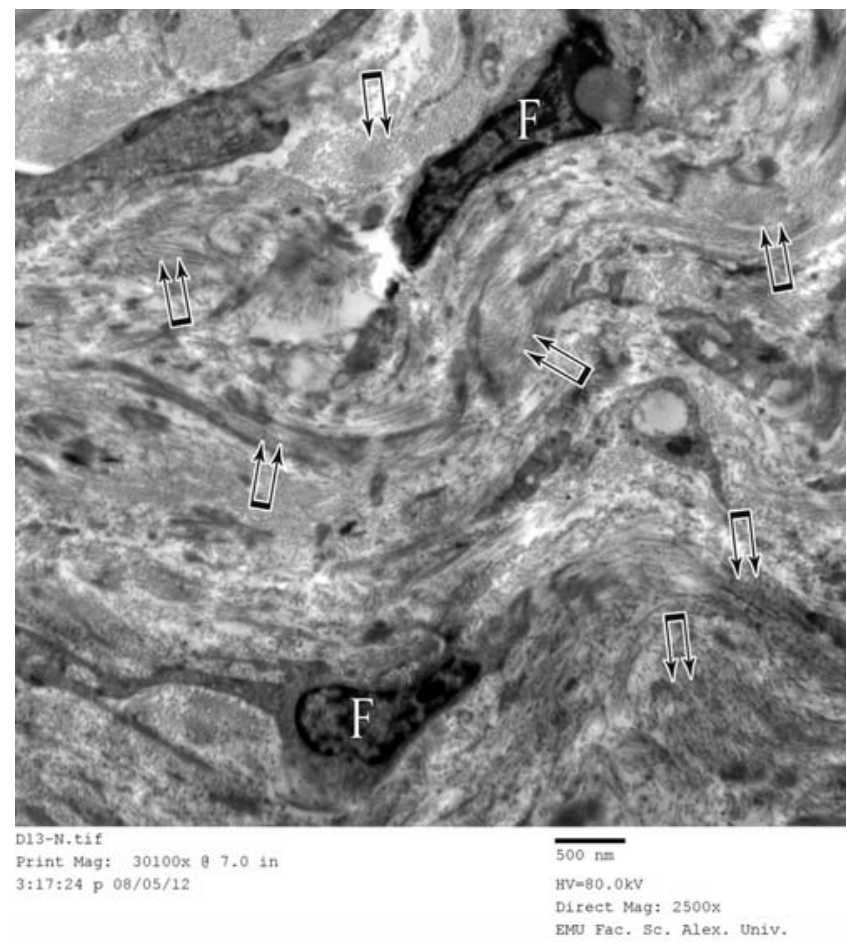

Figure 14. Electron micrograph revealing fibrosis inside a chorionic villus core of a woman with GDM; fibroblasts (F) and collagenous bundles (double arrows) are exceptionally prevalent. Magnification $\times 2500$, Scale bar $=500 \mathrm{~nm}$.

endothelial glucose uptake and glycogen synthesis..$^{[7]}$ Placenta is glucose dependent and the only fetal tissue that can store excess glucose. Moreover, glucose itself activates glycogen synthase and deactivates glycogen phosphorylase that destroys glycogen ${ }^{[1,25]}$ Also, the high affinity glucose transporter 3 (GLUT3) is expressed in the placental endothelium, where it co-localizes with glycogenin, the protein precursor for glycogen synthesis. ${ }^{[1]}$ These areas filled with glycogen may disrupt blood flow and placental exchange by increasing the transfer distance from the maternal blood. ${ }^{[1]}$

Fibroblasts and fibrin deposition were found in the villous core in GDM women in the current study. Gheorman et al., ${ }^{[8]}$ Gauster et al. ${ }^{[23]}$ and Mayhew and Sampson ${ }^{[15]}$ found greater deposition of fibrin in the placenta of diabetic women. The same was described by Meng et al. ${ }^{[2]}$ and Shams et al..$^{[2]]}$ The interruption of blood supply to the placenta causes placental infarcts and fibrosis. Small placental infarcts with minimal fibrosis are considered to be normal at term. However, large placental infarcts and marked fibrosis are associated with 
vascular abnormalities as in GDM. Fibrin deposits may reflect changes in the nature of the villi and this might affect transport of oxygen and nutrients to the fetus. ${ }^{[15,27]}$

The current study revealed the presence of perivillous fibrinoid degeneration in GDM placentas. This was also shown in earlier studies by Jarmuzek et al. ${ }^{[28]}$ and Gabbay-Benziv and Baschat. ${ }^{[2]}$ This fibrinoid degeneration as a special form of necrosis was attributed to the deposition of molecules derived from the hypoxic degenerative processes or blood clotting, or complexes of antigens and antibodies of hypersensitivity reactions together with fibrin that leaked outside the vessels giving the characteristic pink appearance. The fibrinoid may replace the degenerative Sy at the maternofetal exchange surfaces, thus acting as a kind of substitute barrier. Moreover, it is used to adapt the intervillous space to optimized flow conditions to counteract intervillous stasis or turbulence of maternal blood. ${ }^{[2,3,30]}$ Moreover, the present study showed stromal macrophages outside the villous capillaries. Guo et al. ${ }^{[31]}$ showed that these stromal macrophages represented the stromal vascular fraction that was stated in many regeneration processes and diabetes-related complications. Stromal vascular fraction was suggested to achieve healing and regeneration by angiogenesis and extracellular matrix secretion. ${ }^{[31]}$

\section{Conclusion}

This study showed that hyperglycemia and hypoxia are two key factors in the pathophysiologic process of GDM complications, and that it is hyperglycemia that induces hypoxia and oxidative stress in the placenta. Also, the thickened placental barrier, edematous spaces, fibrin deposition and trophoblastic vacuoles in GDM potentially reduce transplacental transport and exchange with aggravation of ischemia and hypoxia.

\section{References}

1. Gül M, Bayat N, Çetin A, Kepekçi RA, Şimşek Y, Kayhan B, Turhan U, Otlu A. Histopathological, ultrastructural and apoptotic changes in diabetic rat placenta. Balkan Med J 2015;32:296-302.

2. Meng Q, Shao L, Luo L, Mu Y, Xu W, Gao C, Gao L, Liu J, Cui Y. Ultrastructure of placenta of gravidas with gestational diabetes mellitus. Obstet Gynecol Int 2015;2015:283124.

3. Aires MB, Dos Santos AC. Effects of maternal diabetes on trophoblast cells. World J Diabetes 2015;6:338-44.

4. Castellucci M, Kaufmann P. Basic structure of the villous trees. In: Benirschke K, Kaufmann P, Baergen RN, editors. Pathology of the human placenta. 5th ed. New York (NY): Springer; 2006. p. 50-120.

5. American Diabetes Association. Standards of medical care in diabetes-2011. Diabetes Care 2011;34:S11-61.

6. Zhang F, Dong L, Zhang CP, Li B, Wen J, Gao W, Sun S, Lv F, Tian H, Tuomilehto J, Qi L, Zhang CL, Yu Z, Yang X, Hu G.
Increasing prevalence of gestational diabetes mellitus in Chinese women from 1999 to 2008. Diabet Med 2011;28:652-7.

7. Desoye G, Hauguel-de Mouzon S. The human placenta in gestational diabetes mellitus. The insulin and cytokine network. Diabetes Care 2007;30 Suppl 2:S120-6.

8. Gheorman L, Pleşea IE, Gheorman V. Histopathological considerations of placenta in pregnancy with diabetes. Rom J Morphol Embryol 2012;53:329-36.

9. Hayat MA. Principles and techniques of electron microscopy: biological applications. 3rd ed. New York (NY): CRC Press; 1989. p. 24-74.

10. Gude NM, Roberts CT, Kalionis B, King RG. Growth and function of the normal human placenta. Thromb Res 2004;114:397-407.

11. Lemasters JJ, Theruvath TP, Zhong Z, Nieminen AL. Mitochondrial calcium and the permeability transition in cell death. Biochim Biophys Acta 2009;1787:1395-401.

12. Pagliarini DJ, Calvo SE, Chang B, Sheth SA, Vafai SB, Ong SE, Walford GA, Sugiana C, Boneh A, Chen WK, Hill DE, Vidal M, Evans JG, Thorburn DR, Carr SA, Mootha VK. A mitochondrial protein compendium elucidates complex I disease biology. Cell 2008; 134:112-23.

13. Slukvin II, Salamat MS, Chandra S. Morphologic studies of the placenta and autopsy findings in neonatal-onset glutaric acidemia type II. Pediatr Dev Pathol 2002;5:315-21.

14. Zorn TM, Zuniga M, Madrid E, Tostes R, Fortes Z, Giachini F, San Martín S. Maternal diabetes affects cell proliferation in developing rat placenta. Histol Histopathol 2011;26:1049-56.

15. Mayhew TM, Sampson C. Maternal diabetes mellitus is associated with altered deposition of fibrin-type fibrinoid at the villous surface in term placentae. Placenta 2003;24:524-31.

16. Joshi M, Kotha SR, Malireddy S, Selvaraju V, Satoskar AR, Palesty A, McFadden DW, Parinandi NL, Maulik N. Conundrum of pathogenesis of diabetic cardiomyopathy: role of vascular endothelial dysfunction, reactive oxygen species, and mitochondria. Mol Cell Biochem 2014;386:233-49.

17. Frohlich JD, Huppertz B, Abuja PM, König J, Desoye G. Oxygen modulates the response of first-trimester trophoblasts to hyperglycemia. Am J Pathol 2012;180:153-64.

18. Jauniaux E, Burton GJ. The role of oxidative stress in placental-related diseases of pregnancy. J Gynecol Obstet Biol Reprod (Paris) 2016; 45:775-85.

19. Magee TR, Ross MG, Wedekind L, Desai M, Kjos S, Belkacemi L. Gestational diabetes mellitus alters apoptotic and inflammatory gene expression of trophobasts from human term placenta. J Diabetes Complications 2014;28:448-59.

20. Kobayashi S. Choose delicately, reuse adequately: the newly recycled process of autophagy. Biol Pharm Bull 2015;38:1098-103.

21. Curtis S, Jones CJ, Garrod A, Hulme CH, Heazell AE. Identification of autophagic vacuoles and regulators of autophagy in villous trophoblast from normal term pregnancies and in fetal growth restriction. J Matern Fetal Neonatal Med 2013;26:339-46.

22. Holmes VA, Young IS, Patterson CC, Pearson DW, Walker JD, Maresh MJ, McCance DR. Optimal glycemic control, pre-eclampsia, and gestational hypertension in women with type 1 diabetes in the diabetes and pre-eclampsia intervention trial. Diabetes Care 2011;34: 1683-8.

23. Gauster M, Desoye G, Tötsch M, Hiden U. The placenta and gestational diabetes mellitus. Curr Diab Rep 2012;12:16-23. 
24. Shapiro F, Mulhern H, Weis MA, Eyre D. Rough endoplasmic reticulum abnormalities in a patient with spondyloepimetaphyseal dysplasia with scoliosis, joint laxity and finger deformities. Ultrastruct Pathol 2006;30:393-400.

25. Yoruk M, Kanter M, Meral I, Agaoglu Z. Localization of glycogen in the placenta and fetal and maternal livers of cadmium-exposed diabetic pregnant rats. Biol Trace Elem Res 2003;96:217-26.

26. Padmanabhan R, Shafiullah M. Intrauterine growth retardation in experimental diabetes: possible role of the placenta. Arch Physiol Biochem 2001;109:260-71.

27. Shams F, Rafique M, Samoo NA, Irfan R. Fibrinoid necrosis and hyalinization observed in normal, diabetic and hypertensive placentae. J Coll Physicians Surg Pak 2012;22:769-72.

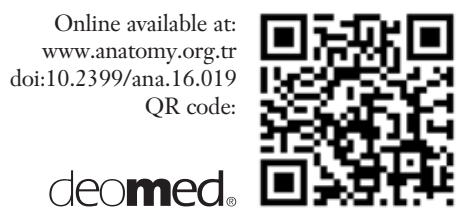

28. Jarmuzek P, Wielgos M, Bomba-Opon D. Placental pathologic changes in gestational diabetes mellitus. Neuro Endocrinol Lett 2015;36:101-5.

29. Gabbay-Benziv R, Baschat AA. Gestational diabetes as one of the "great obstetrical syndromes" - the maternal, placental, and fetal dialog. Best Pract Res Clin Obstet Gynaecol 2015;29:150-5.

30. Augustine G, Pulikkathodi M, Renjith S, Jithesh TK. A study of placental histological changes in gestational diabetes mellitus on account of fetal hypoxia. Int J Med Sci Public Health 2016;5:2457-60.

31. Guo J, Nguyen A, Banyard DA, Fadavi D, Toranto JD, Wirth GA, Paydar KZ, Evans GR, Widgerow AD. Stromal vascular fraction: a regenerative reality? Part 2: mechanism of regenerative action. J Plast Reconstr Aesthet Surg 2016;69:180-8.

Correspondence to: Abdelghany Hassan, MD

Department of Anatomy and Embryology, Faculty of Medicine

Alexandria University, Alexandria, Egypt

Phone: +201115 509509

e-mail: abdelghanyha@yahoo.com

Conflict of interest statement: No conflicts declared.

This is an open access article distributed under the terms of the Creative Commons Attribution-NonCommercial-NoDerivs 3.0 Unported (CC BY-NCND3.0) Licence (http://creativecommons.org/licenses/by-nc-nd/3.0/) which permits unrestricted noncommercial use, distribution, and reproduction in any medium, provided the original work is properly cited. Please cite this article as: Hassan A, Essa TM. Ultrastructure of the placenta in gestational diabetes mellitus. Anatomy 2016;10(3):159-169. 